\title{
Cerebral Venous and Dural Sinus Thrombosis as a Rare Cause of Subarachnoid Haemorrhage in a Patient with Stable Crohn's Disease - A Case Report
}

\author{
Neža Prezelj and Matija Zupan* \\ Department of Neurology, Division of Vascular Neurology, University Medical Centre Ljubljana, 2 Zaloška Street, 1000 Ljubljana, Slovenia
}

Correspondence to:

Matija Zupan, $\mathrm{MD}, \mathrm{PhD}$

Department of Neurology

Division of Vascular Neurology

University Medical Centre Ljubljana

2 Zaloška Street, 1000 Ljubljana, Slovenia

Tel: 0038615223077

E-mail: matija.zupan@kclj.si

Received: December 26, 2016

Accepted: February 28, 2017

Published: March 02, 2017

Citation: Prezelj N, Zupan M. 2017. Cerebral Venous and Dural Sinus Thrombosis as a Rare Cause of Subarachnoid Haemorrhage in a Patient with Stable Crohn's Disease - A Case Report. J Neurol Exp Neurosci 3(1): 11-14.

Copyright: (c) 2017 Prezelj and Zupan. This is an Open Access article distributed under the terms of the Creative Commons Attribution 4.0 International License (CC-BY) (http:// creativecommons.org/licenses/by/4.0/) which permits commercial use, including reproduction, adaptation, and distribution of the article provided the original author and source are credited.

Published by United Scientific Group

\begin{abstract}
Subarachnoid haemorrhage is a known but rare manifestation of cerebral venous and dural sinus thrombosis. We present a case of a 62-year-old female with stable Crohn's disease, who developed thunderclap headache with nausea. Upon presentation, she had no meningism and her neurological examination was normal. Unenhanced computed tomography $(\mathrm{CT})$ revealed a small subarachnoid haemorrhage in the right ambient cistern, extending above the right cerebral convexity. CT angiography did not demonstrate an arterial aneurysm. The patient was admitted to the neurology ward with a working diagnosis of nonaneurysmal subarachnoid hemorrhage. A few days later she developed mild right abducens palsy and blurred vision. Fundoscopy revealed bilateral papilledema. A repeated $\mathrm{CT}$ scan with $\mathrm{CT}$ venography revealed a thrombosis of the transverse and sigmoid sinuses as well as the jugular vein, pronounced particularly on the right side. Thrombophilia testing was positive for activated protein $\mathrm{C}$ resistance in the heterozygous form. We instituted anticoagulation with therapeutic doses of low molecular weight heparin. The clinical course of the thrombosis was favourable. Crohn's disease, especially in combination with inborn thrombophilia, could represent a prothrombotic state. This may be associated with the thrombosis in our patient. In cases of an unusually localised subarachnoid haemorrhage in the absence of an arterial aneurysm, CT venography should be performed to rule out cerebral venous and dural sinus thrombosis.
\end{abstract}

\section{Keywords}

Activated protein $\mathrm{C}$ resistance, Crohn's disease, Cerebral venous and dural sinus thrombosis, Nonaneurysmal subarachnoid haemorrhage, Prothrombotic state, Thunderclap headache

\section{Abbreviations}

CD: Crohn's disease; CT: Computerized brain tomography scan; CTV: Computerized brain tomography venography scan; CVST: Cerebral venous and dural sinus thrombosis; IBD: Inflammatory bowel disease; SAH: Subarachnoid haemorrhage; MRI: brain magnetic resonance imaging scan; MRV: Brain magnetic resonance imaging venography scan

\section{Introduction}

Subarachnoid haemorrhage ( $\mathrm{SAH}$ ) is a rare manifestation of cerebral venous and dural sinus thrombosis (CVST), described in approximately 70 cases in the relevant English literature to date [1]. In general neurological practice, the most common cause of acute non-traumatic $\mathrm{SAH}$ is a ruptured intracranial aneurysm (in $85 \%$ of cases) and less frequently a nonaneurysmal perimesencephalic 
haemorrhage (in $10 \%$ of cases) [2]. Both conditions manifest as SAH primarily at the skull base [2]. The remaining $5 \%$ of causes are associated with a range of uncommon conditions, for instance dural arteriovenous fistulae, vascular malformations, amyloid angiopathy, posterior reversible encephalopathy syndrome, arterial dissection, drug abuse, tumours, vasculitis, pituitary apoplexy, cavernoma bleeding and ultimately CVST $[2,3]$. Therefore, CVST is usually not regarded in the diagnostic workup of spontaneous SAH and can easily be overlooked.

We present a 62-year-old female patient who presented with thunderclap headache. Neurovascular imaging initially showed a small convexal SAH without an arterial aneurysm. After a few days, the patient developed visual disturbances which prompted the search for an alternative diagnosis. Repeated neuroimaging, including cerebral venography, revealed CVST of the transverse and sigmoid sinuses as well as the jugular vein mostly on the right side, upon which the patient could be accurately treated. Our case suggests that neurovascular imaging of patients with $\mathrm{SAH}$ at an unusual location and without an arterial aneurysm should include cerebral venography. Unless treated early with anticoagulation, even in the presence of intracerebral bleeding, CVST can be associated with high mortality $[1,2]$.

\section{Case Report}

A 62-year-old Caucasian female presented to our emergency department with a severe right-sided headache of sudden onset and 3 days duration which had gradually become generalised. The headache started abruptly in a relaxed sitting position. It was initially located in the right ear area, exhibited a dull character and an intensity of 7/10 according to the visual analogue scale. Nausea was an accompanying symptom, but the patient did not report any vomiting or additional neurological symptoms. She had never experienced a similar headache before.

The patient was diagnosed with Crohn's disease (CD) 17 years prior to admission, underwent two resections of the terminal ileum due to intestinal stenosis (the second procedure took place 6 years prior to admission), and had even been considered for biologic treatment due to frequent relapses in the past. Up to admission, the patient had been stable for three months and was not receiving any specific treatment for CD, only supplements of vitamin D. Seven years prior to admission cholecystectomy and the removal of the pancreatic tail due to a papillary tumour had been performed. Over a year ago she had undergone extracorporeal shock wave lithotripsy due to nephrolithiasis. She had been also diagnosed with a left-sided sacroiliitis. The patient did not report allergies, infections or recent trauma. Her family history was notable for ischaemic heart disease and stroke.

Upon arrival at the emergency department, the patient was affected by pain, but her general physical and neurological examination were normal. There was no evidence of meningism.

A non-contrast brain computerized tomographyscan (CT) revealed a small $\mathrm{SAH}$ in the right ambient cistern and over the cerebral convexity in the right temporo-occipital region
(Figure 1). CT angiography was negative for arterial aneurysm. The patient was admitted to the neurological department with a working diagnosis of a small angiographically negative SAH.

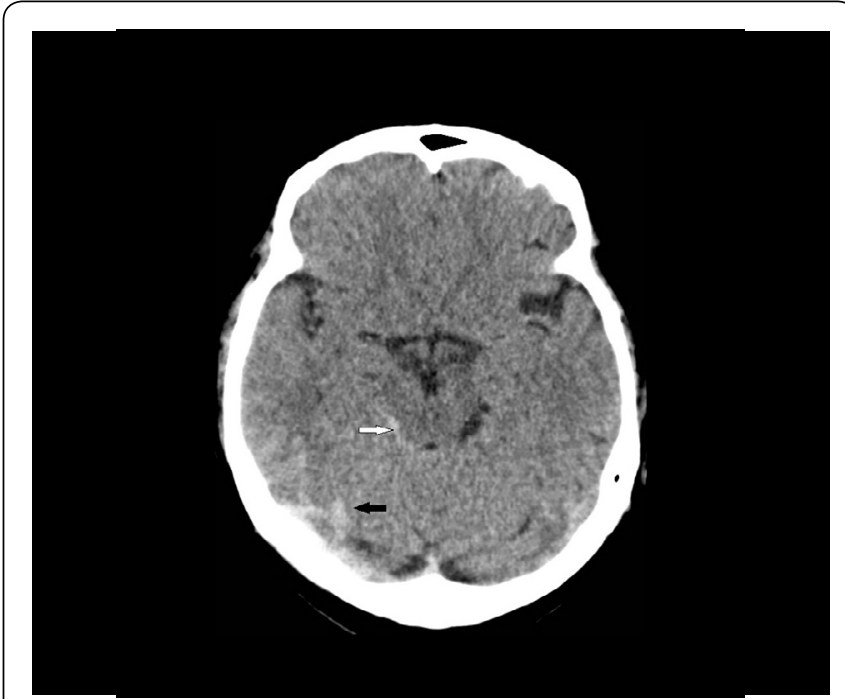

Figure 1: Non-Contrast CT showing blood in the ambient cistern (white arrow) and over the temporo-occipital region (black arrow).

The patient's routine haematological examination including platelet count, haemoglobin, and leukocyte count were normal. Serum biochemistry including liver function tests, glucose, creatinine, urea, potassium, and sodium was also normal, as were tumour markers.

While receiving intravenous analgesic therapy the patient's headache diminished but did not resolve completely. After two weeks she started complaining of blurred vision, declining visual acuity and diplopia. We diagnosed a mild right abducens palsy and bilateral papilledema on fundoscopy. Repeated CT imaging did not demonstrate subarachnoid blood or signs of cerebral venous infarction. Clinical signs of elevated intracranial pressure in addition to the angiographically negative atypical SAH prompted CT venography (CTV), which demonstrated an occlusive thrombosis of the right transverse and sigmoid sinuses as well as jugular vein, and a non-occlusive thrombosis of the left transverse sinus (Figure 2). After establishing the diagnosis of CVST, thrombophilia testing was performed. While heterozygosity for activated protein C resistance was found, testing for antiphospholipid antibodies and lupus anticoagulants was negative. The patient was then started on therapeutic subcutaneous low molecular weight heparin and acetazolamide.

After two weeks, we performed a follow-up brain magnetic resonance imaging (MRI) combined with contrastenhanced MRI venography (MRV). It showed improvement with partial recanalization of the dural sinuses, especially the left transverse sinus, whereas the right transverse and sigmoid sinus were still described as near-occluded (Figure 3).

The patient did not develop clinical signs of inflammatory bowel disease (IBD) relapse during hospitalisation. She reported improvement of vision while ophthalmological supervisions revealed the resolution of papilledema bilaterally. Headaches became less severe and gradually resolved. The 
patient was referred to the urological department due to nephrolithiasis requiring lithotripsy in general anaesthesia. The procedure was successful. Shortly thereafter, long-term peroral anticoagulation with warfarin was initiated. A followup brain MRI performed two months after discharge showed almost complete recanalization of all previously affected dural sinuses and jugular vein. Clinically, the headache and papilledema subsided completely. Occasional outpatient visits were scheduled during the following months.

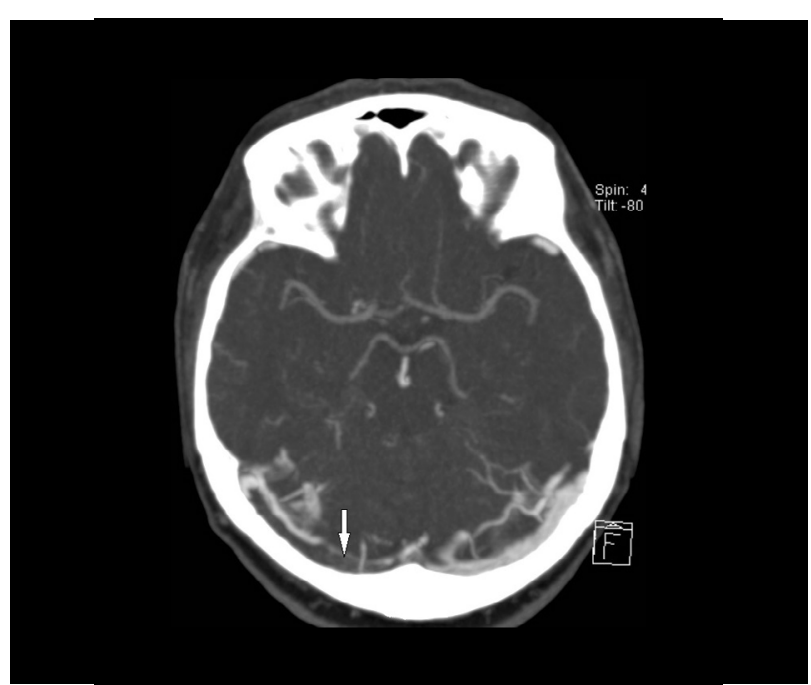

Figure 2: CTV showing signs of dural venous thrombosis in the right transverse sinus (white arrow)

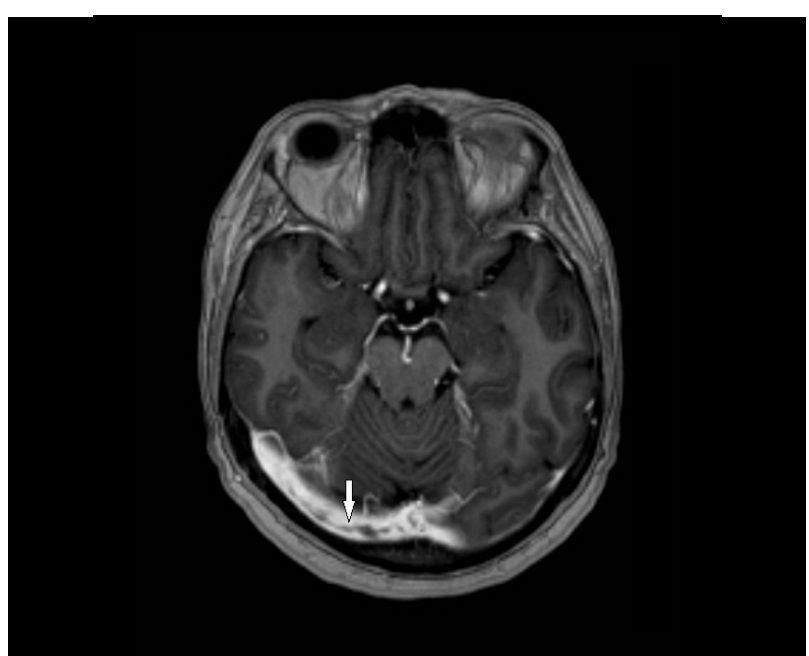

Figure 3: MRV showing partial recanalization of the right transverse sinus (white arrow)

\section{Discussion}

While our case is not a unique presentation of CVST, it highlights the importance of early complete neurovascular imaging including venography when confronted with a patient with thunderclap headache and SAH at unusual locations without an arterial aneurysm. SAH is rarely associated with CVST and as such can be easily overlooked and can be associated with significant consequences. Boukobza et al. reported 22 patients with CVST presenting as isolated $\mathrm{SAH}$ in the absence of parenchymal haemorrhage which suggests that the incidence of concurrent CVST and SAH was $6.4 \%$
[3]. According to cases of CVST reported in literature, $\mathrm{SAH}$ is usually seen at the cerebral convexities adjacent to thrombosed dural sinuses or cortical veins with the absence of blood in the basal cisterns and at the skull base [1]. This was the case in our patient as well. On the other hand, parenchymal haemorrhage is frequently reported in CVST [1] but was absent in our case.

The correct diagnosis of CVST in our case was postponed for about two weeks after the admission, until the patient developed unusual signs and symptoms suggesting elevated intracranial pressure which could not be explained by the small SAH. CVST may be a diagnostic challenge for neurologists as its clinical presentation can be highly variable. Our patient presented with an unusual thunderclap headache which has been reported in about $10 \%$ of patients with CVST [4]. Regarding the frank SAH shown on our patient's initial CT, abrupt headaches should not be surprising. However, the unusual location of nonaneurysmatic SAH and sparing of the basal cisterns should have prompted an initial CTV or even MRI (with a gradient echo sequence) combined with MRV, these two being the gold standard for CVST [5].

The exact pathogenesis of SAH in CVST is not completely understood. Venous parenchymal haemorrhagic infarcts may rupture into the subarachnoid space and cause SAH [6]. In our case, no venous parenchymal infarction was seen on neuroimaging. Alternatively, SAH in CVST could be due to secondary venous hypertension and subsequent rupture of fragile, valveless, thin-walled bridging subarachnoid cortical veins which are devoid of smooth muscle fibres [6, 7]. This is the most plausible explanation for the co-occurrence of $\mathrm{SAH}$ and CVST in our patient. A third hypothesis is that some of the cortical veins can be thrombosed secondary to dural sinus thrombosis with retrograde extension of the thrombus from the affected sinus. This causes hypertension of the veins which dilate and subsequently rupture into the subarachnoid space $[2,6,7]$.

Patients with IBD have a three-fold increased risk of systemic venous thromboembolism compared to the general population. Grainge et al. investigated 13756 patients with IBD and 71672 matched controls, and found that 139 patients and 165 controls developed venous thromboembolism, respectively [8]. Moreover, for patients with IBD, the risk for developing venous thromboembolism was 3.4 times higher compared to controls, and 8.4 times higher at the time of a relapse. The majority of patients with IBD were found to have an active disease at the time of thrombosis [8]. On the contrary, our patient developed CVST during a non-active phase of the disease and did not manifest with a worsening of IBD during the hospitalisation.

In patients with IBD, venous thrombosis usually occurs in the deep veins of the lower extermities and in the pulmonary circulation, less frequently in the portal or mesenteric veins, and in the cerebrovascular system [9]. CVST is rarely described in patients with IBD; furthermore, it more commonly occurs in ulcerative colitis than CD. About 1.3 to $6.4 \%$ of adults with IBD develop CVST at some point during the course of disease $[10,11]$. The underlying mechanisms involved in coagulation activation in patients with IBD remain poorly understood. Coagulation factor abnormalities such as elevated factor VII 
and VIII, increased fibrinogen level, deficiency of antithrombin III, protein C and S have been described in IBD patients [10]. Hereditary thrombogenic mutations such as homozygous C677T mutation in the methylenetetrahydrofolate reductase gene, hyperhomocysteinaemia and factor $\mathrm{V}$ Leiden (FVL) mutation have also been reported $[12,13]$. Guédon et al. reported the prevalence of the heterozygous FVL mutation in thrombotic patients with IBD to be similar to that observed in the non-IBD thrombotic patients, $14.3 \%$ vs. $15.5 \%$, respectively. The prevalence of this mutation in patients with IBD without thrombotic complications and in healthy individuals was much lower, $0 \%$ vs. 3.6\%, respectively [12]. Data from different studies suggest that the FVL mutation is not associated with IBD but, if present, the risk for thrombosis is further increased [12]. The aforementioned hereditary factor $\mathrm{V}$ Leiden mutation is known to be the most common inherited risk factor for thromboembolism and was also positive in our patient in the heterozygous form.

Our patient required long-term anticoagulation with warfarin due to the lasting propensity for thrombotic complications in light of CD and FVL. Fortunately, the patient did not suffer any major adverse effects by postponing anticoagulation treatment. Careful follow-up will be required as $\mathrm{CD}$ and anticoagulation therapy increase the risk of gastrointestinal haemorrhage, as well as due to other challenges of CVST treatment. Although absent in our patient, active gastrointestinal bleeding - not uncommon in the active stage of $\mathrm{CD}$ - is a contraindication to therapeutic anticoagulation. Patients with CD may also exhibit warfarin resistance, either due to the loss of effective surface area from multiple small bowel resections (i.e. "short gut" syndrome) or drug interaction between warfarin and immune modulators, such as 6-mercaptopurine and azathioprine, used in patients, refractory to first-line agents, such as glucocorticoids [14].

\section{Conclusion}

Even to an experienced neurologist, CVST may pose a diagnostic challenge due to a highly variable clinical and, to a lesser extent, neuroradiological presentation. Although CVST is infrequently associated with $\mathrm{SAH}$, the latter may be its sole finding upon initial neuroimaging. We therefore believe a complete initial neurovascular imaging including CTV is warranted in any patient with CT confirmed non-aneurysmal $\mathrm{SAH}$ at an unusual location. While major thromboembolism is not a common manifestation even in the presence of IBD, a history of IBD, particularly in active state, should be taken into consideration in evaluating these patients with acute headache.

\section{Acknowledgments}

The authors express their gratitude to Ina Živanović, MD, for professional English editing and critical supervision of the manuscript.

\section{Funding Details}

We have not received any financial support for this work.

\section{Conflict of Interest}

The authors have no conflicts of interest or any sources of funding to report. We certify that the submission is original work and is not under review at any other publication.

\section{Informed Consent from the Patient}

Approved (signed) informed consent for publication of her details and images was obtained from the patient.

\section{References}

1. Sahin N, Solak A, Genc B, Bilgic N. 2014. Cerebral venous thrombosis as a rare cause of subarachnoid haemorrhage: case report and literature review. Clin Imaging 38(4): 373-379. https://doi.org/10.1016/j. clinimag.2014.03.005

2. Panda S, Prashantha DK, Shankara SR, Nagaraja D. 2010. Localized convexity subarachnoid haemorrhage - a sign of early cerebral venous sinus thrombosis. Eur J Neurol 17(10): 1249-1258. https://doi. org/10.1111/j.1468-1331.2010.03001.x

3. Boukobza M, Crassard I, Bousser MG, Chabriat H. 2016. Radiological findings in cerebral venous thrombosis presenting as subarachnoid haemorrhage: a series of 22 cases. Neuroradiology 58(1): 11-16. https:// doi.org/10.1007/s00234-015-1594-5

4. De Bruijn SF, Stam J, Kappelle LJ. 1996. Thunderclap headache as first symptom of cerebral venous sinus thrombosis. Lancet 348(9042): 16231625. https://doi.org/10.1016/S0140-6736(96)07294-7

5. Hassan A, Ahmad B, Ahmed Z, Al-Quliti KW. 2015. Acute subarachnoid haemorrhage. An unusual clinical presentation of cerebral venous sinus thrombosis. Neurosciences 20(1): 61-64.

6. Oppenheim C, Domigo V, Gauvrit JY, Lamy C, Mackowiak-Cordoliani MA, et al. 2005. Subarachnoid haemorrhage as the initial presentation of dural sinus thrombosis. Am J Neuroradiol 26(3): 614-617.

7. Arévalo-Lorido JC, Carretero-Gómez J. 2015. Cerebral venous thrombosis with subarachnoid haemorrhage: a case report. Clin Med Res 13(1): 40-43. https://doi.org/10.3121/cmr.2014.1225

8. Grainge MJ, West J, Card TR. 2010. Venous thromboembolism during active disease and remission in inflammatory bowel disease: a cohort study. Lancet 375(9715): 657-663. https://doi.org/10.1016/S01406736(09)61963-2

9. Papa A, Gerardi V, Marzo M, Felice C, Rapaccini GL, et al. 2014. Venous thromboembolism in patients with inflammatory bowel disease: focus on prevention and treatment. World J Gastroenterol 20(12): 31733179. https://doi.org/10.3748/wjg.v20.i12.3173

10. Katsanos AH, Katsanos KH, Kosmidou M, Giannopoulos S, Kyritsis AP, et al. 2013. Cerebral sinus venous thrombosis in inflammatory bowel diseases. QJM 106(5): 401-413. https://doi.org/10.1093/qjmed/ hes 229

11. Kim I, Min KH, Yeo M, Kim JS, Lee SH, et al. 2015. Unusual case of cerebral venous thrombosis in patient with Crohn's disease. Case Rep Neurol 7(2): 115-120. https://doi.org/10.1159/000430805

12. Guédon C, Le Cam-Duche V, Lalaude O, Ménard JF, Lerebours E, et al. 2001. Prothrombotic inherited abnormalities other than factor $\mathrm{V}$ leiden mutation do not play a role in venous thrombosis in inflammatory bowel disease. Am J Gastroenterol 96(5): 1448-1454. https://doi.org/10.1111/j.1572-0241.2001.03797.x

13. Papa A, Danese S, Grillo A, Gasbarrini G, Gasbarrini A. 2003. Review article: inherited thrombophilia in inflammatory bowel disease. $\mathrm{Am}$ $J$ Gastroenterol 98(6): 1247-1251. https://doi.org/10.1111/j.15720241.2003.07491.x

14. Kwon Y, Koene RJ, Cho Y. 2016. A case of cerebral venous sinus thrombosis associated with Crohn's disease: dilemma in management. Gastroenterol Rep (Oxf) 4(2): 154-157. https://doi.org/10.1093/gastro/ gou079 